\title{
Modelo de gestión de capital humano para mejorar el clima organizacional en la empresa Petrocasa
}

\author{
Human capital management model to improve the organizational climate in the \\ Petrocasa company
}

\author{
Modelo de gestão de capital humano para melhorar o clima \\ organizacional na empresa Petrocasa
}

Recibido: octubre 2019

Arbitrado: noviembre 2019

Publicado: enero 2020
《 Wilmer Rodríguez

wilmerodriguez22@gmail.com

ORCID: 0000-0002-5393-3758

Universidad de Carabobo, Venezuela
RESUMEN

La investigación tiene como objetivo plantear un Modelo de gestión de capital humano para mejorar el clima organizacional en la empresa Petrocasa. Metodológicamente la investigación se basa en una investigación descriptiva, no experimental, de campo, modalidad proyecto factible, por la cual se utilizaron como técnicas la encuesta, la entrevista y la observación participante, los instrumentos de recolección de datos son el cuestionario, la guía de entrevista estructurada y las notas de campo. La muestra es de tipo probabilística estratificada, constituida por 30 trabajadores del departamento de ventas de la Gerencia de Comercialización y Logística de la empresa caso estudio. El cuestionario será aplicado a los trabajadores y la entrevista a la Dirección. De esta manera se pudo evidenciar que la empresa carece de estrategias motivacionales. Por consiguiente, es factible plantear estrategias que permitan mejorar el clima organizacional de la empresa o que permitirá potencializar la efectividad y productividad de los trabajadores.

Palabras clave: Ambiente laboral; capital humano; clima organizacional; deserción; estrategias, modelo de gestión
The objective of the research is to propose a Human capital management model to improve the organizational climate in the Petrocasa company. Methodologically the research is based on a descriptive, nonexperimental, field research, feasible project modality, by which the survey, interview and participant observation were used as techniques, the data collection instruments are the questionnaire, the structured interview and field notes. The sample is of stratified probabilistic type, consisting of 30 workers from the sales department of the Marketing and Logistics Management of the case study company. The questionnaire will be applied to the workers and the interview to the Management. In this way it was possible to show that the company lacks motivational strategies. Therefore, it is feasible to propose strategies that improve the organizational climate of the company or that will enhance the effectiveness and productivity of workers.

Key words: Work environment; human capital organizational climate; desertion; strategies, management model
RESUMO

O objetivo da pesquisa é propor um Modelo de gestão do capital humano para melhorar o clima organizacional na empresa Petrocasa. Metodologicamente a pesquisa é baseada em uma modalidade descritiva, não experimental, pesquisa de campo, projeto viável, pela qual foram utilizadas como técnicas o levantamento, a entrevista e a observação participante, os instrumentos de coleta de dados são o questionário, a entrevista estruturada e notas de campo. A amostra é do tipo probabilística estratificada, composta por 30 trabalhadores do departamento de vendas da Gerência de Marketing e Logística da empresa do estudo de caso. O questionário será aplicado aos trabalhadores e a entrevista à Direção. Desta forma, foi possível mostrar que a empresa carece de estratégias motivacionais. Portanto, é viável propor estratégias para melhorar o clima organizacional da empresa ou para aumentar a eficácia e a produtividade dos trabalhadores.

Palavras chave: Ambiente de trabalho; capital humano; clima organizacional; deserção; estratégias, modelo de gestão 


\section{INTRODUCCIÓN}

$\mathrm{L}$ a gestión de capital humano es determinante para garantizar que las organizaciones permanezcan sanas en el tiempo, en la actualidad ha adquirido una función aún más importante en la creación de ventajas competitivas para una empresa ya que se la ha enfocado en la búsqueda, atracción, selección y capacitación de los trabajadores idóneos para una organización, siendo la clave del éxito de una organización el desarrollo de un conjunto de competencias que la distinguen de las demás, las cuales proporciona una base a largo plazo para realizar las innovaciones, cambios, desarrollo de productos y servicios, para finalmente cumplir con la misión empresarial.

Además, las condiciones sociales, económicas y culturales de este nuevo siglo, como el desarrollo de los sistemas de protección social, acaba el colonialismo, equilibrio entre superpotencias que caracterizó la "guerra fría", desaparición de los estados comunistas europeos, crisis de la economía mixta y la ofensiva neoliberal, mundialización creciente de la economía-mundo y la crisis de identidad del estado-nación, la nueva división del trabajo, progreso impetuoso de la urbanización del mundo, entre otros (Gutiérrez, 2012:1), hacen imprescindible que las empresas sean altamente creativas, innovadoras, se adapten ágilmente a los cambios, sobrevivan y crezcan, y para lograrlo necesariamente deben contar con una fuerza laboral comprometida, capacitada y motivada, que trabaje en busca del logro de la misión de la organización, pero que a la vez sienta que está logrando satisfacer sus deseos, expectativas y necesidades más profundas.

En la actualidad, las actividades organizacionales presentan a las empresas un desafío competitivo sin precedentes, donde es importante plantearse como misión de la organización el ser y lucir atractiva para clientes, especialmente para su cliente interno, quienes llevarán a la empresa al éxito a largo plazo. Para lograr el desarrollo de una empresa, se debe conocer y conducir el comportamiento de su gente.

Es así como, el comportamiento del ser humano en general, no es errático, sino que responde a ciertos patrones o estructuras que suelen agruparse bajo el concepto de personalidad; inclusive es posible afirmar, que la mejor forma de predecir el comportamiento de una persona en el futuro es analizar su comportamiento en el pasado. Si bien, es posible crecer, desarrollarse y modificar comportamientos, para aprender, en el caso, es de vital importancia entonces, facilitar las herramientas necesarias, para capacitar las competencias requeridas para un puesto, las cuales varían con el tiempo y son distintas para una posición en diferentes organizaciones.

En la actualidad a nivel mundial las tendencias y perspectivas de la gestión de talento humano se imponen, las cuales a su manera ayudan al incremento y desarrollo de la competitividad y productividad, pretendiendo optimizar la gestión del personal de la empresa. Los profesionales de Gestión Humana deben enfrentarse, hoy más que nunca, a un contexto donde el cambio se ha vuelto una constante y donde las compañías que no logren situarse un paso adelante respecto de 
las tendencias locales y globales, les costará mucho mantener su liderazgo y posicionamiento en el mercado. Según Vivar (2014):

Hace 50 años las empresas requerían el talento de unos pocos para que pongan su creatividad e iniciativa en juego; estas personas constituían la cima de la pirámide: los directivos o gerentes de la compañía. El resto de los trabajadores sólo tenían que cumplir las tareas definidas y ejecutar órdenes o seguir procesos mecánicamente en base a instructivos pre establecidos y que no podían ser modificados. Pero la competencia se incrementó, los clientes se hicieron cada vez más exigentes y los intangibles comenzaron a cobrar cada vez más valor. Es allí cuando surge la necesidad en las empresas de crear valor en todo su personal, potenciando el rol de los líderes y empoderando a toda su gente, en todos los niveles organizacionales. (p.15).

La gestión de talento humano permite el desarrollo integral de las personas ya sea de forma individual o en grupo para alcanzar el éxito empresarial. Una de las tendencias más relevantes en la actualidad se refiere a discursos que pretenden rescatar al ser humano dentro de la empresa, para lo cual esto no se convierte en los procesos de la gestión de capital humano que utilizan las organizaciones nacionales e internacionales. Con respecto a esto, Vivar (2014) afirma que es necesario "agregar valor al negocio mediante una gestión que equilibre nuestra preocupación por la motivación, el crecimiento y desarrollo de nuestra gente y la conjugue con los grandes objetivos de nuestras compañías: rentabilidad, crecimiento y liderazgo en el mercado" (p.1).

Los factores de competitividad que deben intervenir en la permanencia de la empresa en un mercado globalizado es la introducción de ciencia y tecnología, generación de valor agregado, asociatividad, y la capacidad de internacionalizarse, además un adecuado manejo de la administración y gestión de todo lo referente al talento humano que en definitiva es el eje central de una empresa, dichos factores deben ser manejados adecuadamente para conseguir posicionarnos en el mercado y llegar a ser competitivos. (Saldarriaga, 2008)

Los nuevos desafíos en el ámbito empresarial, como la globalización, las nuevas tecnologías de la comunicación, las normas de calidad, el marketing 3.0, entre otras, y la complejidad de los entornos sociales, laborales y culturales, donde se destacan la movilidad laboral y social, las nuevas formas de trabajo, el auge de la economía informal y responsabilidad social empresarial, permiten a las personas desarrollar nuevas competencias y actualizar las que poseen, o simplemente desechar algunas porque ya son obsoletas. Una respuesta efectiva a estos desafíos es la preparación y la ayuda a las personas con el fin de que desarrollen competencias necesarias para que puedan realizar un buen desempeño personal y profesional.

En relación a lo expuesto, para el logro de mejoras competitivas en la organización, es indispensable contar con un recurso humano dispuesto a ir de la mano con los objetivos organizacionales. Es por ello, que el recurso humano juega un papel importante, y en la medida en que los trabajadores se sientan a gusto en su ambiente laboral, favorecidos en su desarrollo 
profesional, salud física, y psicológica, serán eficientes, pertinentes, y efectivos en el desarrollo de las tareas relacionadas con sus puestos de trabajo, lo que hará organizaciones más competitivas. En este sentido, Herrera (2011), afirma que "Las personas son consideradas recursos a partir de que aparecen en la empresa como elementos de trabajo, donde utilizan y transforman otros recursos" (p.3) Por su parte, Chávez y Vizcaíno (2017:1), señala que "las personas constituyen un recurso valioso dentro de la organización y la formación del talento humano es una variable determinante para lograr la competitividad de las organizaciones".

De esta forma, al estudiar el Clima Organizacional se obtiene información sobre los valores, actitudes y creencias de las personas que la componen, y se genera retroalimentación acerca de los procesos que determinan los comportamientos de la organización, lo que permite generar cambios programados en la estructura organizacional, conducta y satisfacción de sus miembros. Además, permite conocer la percepción que los trabajadores tienen de la empresa y su ambiente de trabajo, logrando así identificar los principales factores que impiden la creación de un ambiente propicio para la organización.

De acuerdo a lo planteado anteriormente, al presentar la realidad del escenario venezolano, como la actual situación económica, política de gobierno, políticas salariales, falta de liderazgo, comunicación, y compromiso por parte de líderes, entre otros factores, se ha generado un ambiente poco propicio para el desarrollo de las empresas, afectando el clima en las organizaciones, tanto públicas como privadas, lo cual ha traído como consecuencia la desmotivación del personal, incumplimiento de las políticas y metas de las organización, ausentismo, falta de compromiso por parte del empleado, bajo rendimiento, improductividad, inconformidad, entre otros .

Sin embargo, las empresas venezolanas, han funcionado por mucho tiempo manejando escenarios difíciles, sobreviviendo dentro de grandes cambios y transformaciones, dando poca importancia al estudio y comprensión del Clima Organizacional y de su comportamiento en pro de mejorar su eficiencia y competitividad. El mismo, es un factor difícil de cuantificar, pero es determinante para el éxito de las organizaciones y considerando además que las organizaciones forman parte importante del motor económico del país, su estudio debe ser notablemente considerado por este tipo de empresas.

Por su parte, Chiavenato (2009) señala que el Clima Organizacional dado en el ambiente interno entre los miembros de la organización, está estrechamente ligado al grado de motivación de los empleados, aseverando que cuando existe un alto nivel de motivación, el Clima motivacional permite desarrollar relaciones satisfactorias de colaboración, interés, compromiso, entre otros. Mientras que al existir bajos niveles de motivación tienden a desarrollar desinterés, apatía, descontento, depresión, entre otros, demostrando que un personal desmotivado no contribuye con los logros de los objetivos organizacionales 
Por ello, es necesario establecer modelos de gestión de capital humano para contrarrestar los efectos negativos y generar ventajas competitivas, basadas en las debilidades presentes en las empresas en lo referente al Clima Organizacional, para proporcionar herramientas claves y lograr en las empresas el cambio necesario para incursionar positivamente en su mercado de interés. Al desarrollar ventajas competitivas se establece un diferencial en la organización con respecto a las empresas del mismo rubro.

Según Moreno (2008), estudios previamente realizados establecen que el clima organizacional en las empresas del estado Carabobo es negativo, manifestándose improductividad, temor, inseguridad, desmotivación y riesgos que deben ser tomados en cuenta por las gerencias para la supervivencia de las empresas, estando dentro de las posibles causas la falta de liderazgo, deficiencia o ausencia de objetivos definidos para las empresas, carencia de planes estratégicos, entre otros.

Dichos síntomas evidenciados en estudios anteriores, representan una alerta para las organizaciones del sector y sus líderes. Es a través del estudio y mejora del Clima Organizacional que se busca que los trabajadores se sientan cómodos en su ambiente de trabajo, y logren una percepción favorable que les permita desarrollar sus labores con alta eficiencia y rendimiento, impactando positivamente en la competitividad del sector, como es el caso de Petrocasa, S.A, empresa de interés social del Estado venezolano ubicada en la carretera nacional Valencia-Los Guayos, en Valencia, estado Carabobo, que cuenta con un proceso industrializado en la fabricación de elementos de construcción elaborados con Policloruro de Vinilo (PVC) de alta tecnología que constituye un sistema productivo para la mejora de la vivienda y el hábitat que brinda seguridad social y estabilidad a los ciudadanos.

Actualmente, se percibe en los empleados de la empresa Petrocasa, S.A. la ausencia de los elementos que abarca el clima organizacional, tales como: comunicación, motivación, estructura, relaciones, conflicto, identidad, liderazgo y comprensión; lo que llama la atención puesto que forman parte de los componentes del clima organizacional, teniendo en cuenta que un ambiente laboral ameno, con un clima laboral que promueva el sentido de pertenencia y el compromiso con las tareas, será siempre una ventaja competitiva para las empresas, ya que la calidad, la productividad y el mejor desempeño de los trabajadores, tiene una relación directa con el ambiente que gira a su alrededor.

Además, la gerencia no conoce cuales son los factores críticos que influye en la deserción del talento humano es su entorno laboral, que genera niveles de insatisfacción laboral. Naturalmente si los directivos de manera continua monitorean los niveles de satisfacción de su personal a través de los factores del clima organizacional, no se tomarían decisiones erradas que provoquen la deserción de un talento, el cual repercute en la rentabilidad de la empresa. De allí, surge la necesidad de desarrollar un Modelo de gestión de capital humano para mejorar el clima organizacional en la empresa Petrocasa, S.A. 
Lo que permitiría proporciona herramientas claves para lograr en las empresas el cambio necesario para incursionar positivamente en su mercado de interés. La implementación de un Modelo de gestión de capital humano para mejorar el clima organizacional en la empresa Petrocasa, S.A. establecerá las estrategias necesarias para lograr la permanencia en el sector construcción, desarrollando y aplicando ventajas competitivas que le ayuden a enfrentar con éxito y siempre estando alerta, los cambios que se producen en el entorno.

Con el modelo de gestión de capital humano se busca que los recursos humanos se sientan cómodos y motivados en su ambiente de trabajo, y logren una percepción favorable que les permita desarrollar plenamente sus habilidades, compromiso, labores con alta eficiencia y rendimiento. A su vez, se sientan identificados y plenamente satisfechos con la organización y sus objetivos, siempre con el propósito de generar el máximo bienestar físico y mental de los trabajadores. Otro factor importante es la satisfacción del cliente interno, y con el desarrollo de las estrategias se busca mejorar la calidad de los productos o servicios que brindan las empresas del sector construcción para permanecer en los mercados de interés.

\section{MÉTODO}

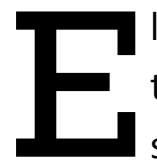

I diseño metodológico se basa en una investigación de tipo de campo, dado que este tipo de estudio se desarrolló para conocer lo que sucede en lo relacionado con una situación o modelo empírico, en función de establecer las razones de su ocurrencia, influencia o impacto sobre otros objetos, sujetos o situación. Descriptiva ya que se describen las características del fenómeno tal como se presentan en la realidad, interpretando correctamente los eventos y situaciones originadas.

El diseño dada las características propias, presentes en la realidad que se estudia, la investigación se desarrolló siguiendo los lineamientos de la modalidad de Proyecto Factible, ya que, su propósito fundamental consiste en dar solución eficaz, pronta y oportuna a un problema de tipo práctico que persiste en el clima organizacional del capital humano en la empresa Petrocasa S.A. con la finalidad de detectar los problemas existente en el mismo y generar propuestas que permitan resolver algún problema práctico.

Para el desarrollo de la investigación la población fue constituida por directivos, empleados medios y base que laboran en la empresa Petrocasa, S.A. La muestra es tipo probabilística dado que la población en subconjuntos posea características comunes.

Para dicha investigación, se empleó como técnica de recolección de datos la encuesta, permitiendo obtener información de gran importancia. Con respecto a la encuesta, Tamayo y Tamayo (2007), señala que "es aquella que permite dar respuestas a problemas en términos descriptivos como de relación de variables, tras la recogida sistemática de información según un diseño previamente establecido que asegure el rigor de la información obtenida" (p.124). 
Como instrumento de recolección de datos se usó el cuestionario, definido por Tamayo y Tamayo (ob. cit.), como aquel que "contiene los aspectos del fenómeno que se consideran esenciales; permite, además, aislar ciertos problemas que nos interesan principalmente; reduce la realidad a cierto número de datos esenciales y precisa el objeto de estudio" (p.124). Por lo tanto, el cuestionario estará estructurado por una serie de preguntas cerradas con alternativas de respuesta dicotómicas en donde el encuestado seleccionará una sola opción de acuerdo a su opinión.

A fin de recabar la información necesaria en la investigación, se utilizó una entrevista estructurada, está se caracteriza por ser realizada a partir de una guía de entrevista previamente elaborada que se aplica de forma sistémica, tanto en el contenido de las preguntas como en su orden. Tiene la ventaja de disminuir las desviaciones del entrevistador. Según lo planteado por Tamayo y Tamayo (2007), la entrevista estructurada se define como "la relación directa establecida entre el investigador y su objetivo de estudio a través de individuos o grupos, con el fin de obtener testimonios reales".

La validación del instrumento se obtuvo a través del juicio de expertos, actividad que se revisó en todas las fases de la investigación, a fin de someter el modelo a la consideración y juicio de conocedores de la materia en cuanto a gerencia y metodología se refiere y así facilitar el montaje metodológico del instrumento tanto de forma como de fondo, con el fin único de su evaluación y al considerar la misma, hacer las correcciones que tuvieran lugar, para de esta forma garantizar la calidad y certidumbre del modelo.

De acuerdo a las características de los instrumentos, la confiabilidad se determinó mediante la aplicación de Kuder-Richardson, para la encuesta aplicada a los trabajadores de la empresa caso estudio.

\section{RESULTADO Y DISCUSIÓN}

$\mathrm{U}$ na vez aplicado el instrumento y realizado un análisis de la situación actual de la empresa Petrocasa. S.A se pudo apreciar la carencia de factores motivacionales, intrínsecos y extrínsecos que afectan de una u otra forma el clima organizacional y conllevan a la desmotivación de los trabajadores, entre los que destacan inconformidad con el salario que perciben, no son recompensados o reconocidos por su trabajo en la empresa, sus aportes o sugerencias no son tomadas en cuenta, consideran que no obtienen el máximo provecho de sus capacidades, habilidades y potencial personal.

Todo lo señalado anteriormente, trae como consecuencia la fuga de talento humano, bajo desempeño laboral, lo que a su vez no permite que la empresa obtenga mejores resultados.

Sin embargo, para que exista un buen desempeño laboral es muy importante que los trabajadores entiendan cuáles son sus funciones o tareas específicas, los procedimientos que se deben seguir, las políticas que se deben respetar, los objetivos que deben cumplir. Una buena forma de mejorar el desempeño laboral de los trabajadores es motivándolos constantemente, y para ello podemos utilizar diferentes técnicas como pueden ser delegarles una mayor autoridad, 
darles mayores responsabilidades, recompensar los logros obtenidos, ofrecer un buen clima laboral.

\section{Propuesta de un modelo de gestión de capital humano para mejorar el clima organizacional en la empresa Petrocasa, S.A}

Actualmente son pocas las empresas que aplican la motivación para el bienestar y/o satisfacción laboral de sus trabajadores y el incremento de su productividad, dado que muchas de ellas ven esto como un gasto y no como una inversión para el crecimiento de la misma, es por ellos que muy pocas empresas conservan a su personal por tiempos prolongados, es decir, aquellas que les proporcionan algún tipo de motivador a sus trabajadores se ve reflejado en actitud positiva que éste toma ciertas circunstancias laborales y tienden a prolongar su estancia.

Siendo este el caso de la empresa Petrocasa. S.A. la cual se enfocan únicamente a que el trabajo se realice de forma correcta, no importando como lo realice y como se siente el trabajador ante ciertas circunstancias en el proceso, es aquí un punto importante donde las empresas deben de tomar en cuenta al trabajador como un elemento importante en el desarrollo y termino de la operación, ya que una persona bien motivada realizara su labor de manera satisfactoria.

Es por ello que se presenta un Modelo de gestión de capital humano para mejorar el clima organizacional en la empresa Petrocasa, S.A., constituye una herramienta gerencial para esta organización, que permita el desempeño y la eficiencia laboral, así como mantener los niveles de satisfacción del capital humano, los cuales participan en las labores de planificación, organización, dirección y control, todas ellas propias del proceso administrativo. A través del plan estratégico se fomentarán las capacidades o competencias requeridas para el desempeño laboral efectivo de las funciones asignadas al personal.

El modelo de gestión hace referencia a los objetivos y metas que a través de éste se persigue, de modo que se formulen los objetivos. Asimismo, se describirán las estrategias y acciones a considerarse para mejorar el clima organizacional, estimular el desempeño laboral y la satisfacción en el personal que labora en la empresa.

\section{Fundamentación de la propuesta}

El modelo de gestión de capital humano se fundamenta en las teorías de la motivación: Teoría de Motivación Humana de Maslow y la Teoría Situacional de Hersey y Blanchard. La teoría de Maslow se fundamenta bajo la hipótesis de que dentro de cada individuo o sujeto, existe una jerarquía de cinco necesidades entre estas se pueden encontrar: Fisiológicas (hambre, sed, refugio, y otras necesidades corporales del individuo), Seguridad (cuidado y protección contra los daños físicos y emocionales), Sociales (sentido por pertenencia, aceptación), Estima (autonomía, logro estatus, reconocimiento, atención) y Autorrealización (crecimiento y desarrollo del propio potencial). 
Un factor importante que se debe saber es que las necesidades no se van a satisfacer por completo, y saber que aquella que alcanza un nivel deja de motivar, si la misma está bastante complacida, la siguiente se vuelve dominante. Según Maslow (1954) si se desea motivar a un individuo se necesita entender y saber en qué nivel de la jerarquía se encuentra esa persona y concentrarse en el nivel de esta satisfacción.

En cuanto a la teoría de Hersey y Blanchard, tiene la premisa de que la relación del liderazgo de un colaborador con el trabajo es fundamental y que la actitud de alguien hacia su puesto de trabajo muy bien podría determinar el éxito o el fracaso. Se basa en mantener un equilibrio entre dos tipos de comportamiento que ejerce un líder para adaptarse al nivel de desarrollo de su equipo de trabajo. Por consiguiente, el gerente que desarrolle a sus subordinados, aumente su confianza y les ayude a aprender su trabajo, logrará que los objetivos se logren con mayor eficacia.

\section{Desarrollo del modelo de gestión de capital humano}

\section{Descripción del modelo de gestión del talento humano}

Es necesario considerar la presentación del modelo de gestión de capital humano para mejorar el clima organizacional del personal de la empresa Petrocasa, S.A. El modelo tiene siete dimensiones que están distribuidas en dos partes descritas en variables internas y variables externas del trabajador.

El modelo contempla una secuencia del comportamiento de las variables de estudio el cual los directivos de recursos humanos deben considerar para alcanzar la eficiencia organizacional. La secuencia se visualiza por cada arco con sentido y dirección a la variable afectada el cual se explica a continuación.

- Condiciones de Autorrealización e involucramiento laboral. Como punto de partida se deben considerar las Condiciones Laborales del trabajador; en esta dimensión se considera la estabilidad laboral y la economía hacia el factor humano ya que es una necesidad básica en el ser humano que de acuerdo al nivel del sueldo genera un estilo de vida diferente. La dimensión o variable de condiciones laborales tiene un efecto directo sobre el involucramiento de los trabajadores.

- Supervisión e Involucramiento laboral. Se observa que la dimensión de supervisión considera la relación entre los trabajadores y el jefe inmediato superior que puede ser favorable o desfavorable para que el colaborador se pueda involucrar en los objetivos de la organización.

- Supervisión y comunicación. Se observa que la dimensión de supervisión considera la relación entre los trabajadores y el jefe inmediato superior que puede ser favorable o desfavorable para la comunicación de los mismos. 
- Comunicación e involucramiento laboral. También se observa que la dimensión de comunicación considera la fluidez de la transferencia de la información que existe en la organización. La comunicación contempla la cantidad de información que se transmite entre los trabajadores, y entre los trabajadores y jefes. Se contempla la forma como se entrega la información. La comunicación puede ser favorable o desfavorable para que los trabajadores puedan involucrarse o no.

- Involucramiento laboral y realización personal. La dimensión de involucramiento laboral, que considera la entrega y responsabilidad de los trabajadores para con las tareas de la organización puede ser favorable o desfavorable para la realización personal.

- Realización personal y factores higiénicos. La dimensión de la realización personal, que considera sentido de superación y utilidad social de los trabajadores dela organización y que este puede ser favorable o desfavorable para los factores higiénicos.

- Factores higiénicos y eficiencia organizacional. La dimensión de los factores higiénicos, que considera los motivadores y desmotivadores de los trabajadores de la organización y que este puede ser favorable o desfavorable para la eficiencia organizativa.

\section{Etapas de la implementación del modelo}

La gestión del capital humano es un modelo que se instala a través de un programa que contempla las siguientes etapas que se suceden de la siguiente manera:

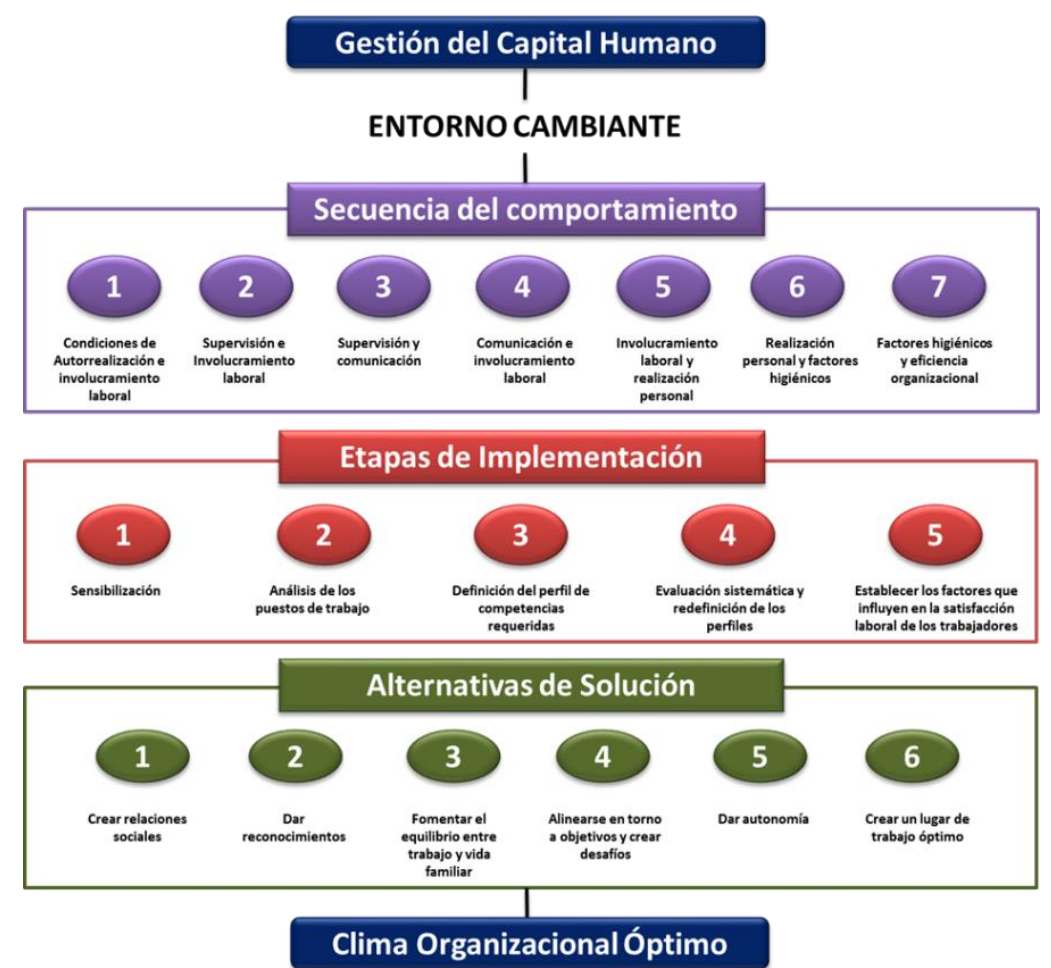

Figura 1. Modelo de Gestión de Capital Humano. 


\section{CONCLUSIÓN}

$\mathrm{L}$ uego de procesar la información a través de una revisión bibliográfica y el correspondiente análisis de los resultados obtenidos se ha llegado a las conclusiones enunciadas a continuación:

- La empresa carecía de estrategias motivacionales.

- Se evidencio inconformidad con el salario que perciben, no son recompensados o reconocidos por su trabajo en la empresa.

- Se pudo observar que los trabajadores no son recompensados o reconocidos por su trabajo en la empresa; aunque señalan que les asignan responsabilidades importantes que lo motivan a trabajar.

- Los trabajadores aseguran que sus aportes o sugerencias no son tomadas en cuenta, la mayoría de los trabajadores se consideran tener habilidades para el liderazgo y la comunicación dentro en la empresa permite la integración del personal que en ella laboral.

Se debe implementar el modelo propuesto, con el fin de impulsar lo más pronto posible las medidas estipuladas para mejorar el clima organizacional en la organización y la satisfacción laboral de los trabajadores. Seguidamente, se debe implementar la figura de un buzón de sugerencias para que el personal puedan plantear actividades o tópicos que resulten de su interés, para que al cabo de cierto período sean analizadas y se elijan aquellas que combinen estratégicamente los conocimientos, técnicas y relaciones interpersonales, con el objeto de contar con un talento humano integral.

Por otro lado, se debe establecer un ambiente de trabajo que propicie mayor participación y cooperación de todos los miembros de la empresa. Reconocer a los trabajadores por su buen desempeño dentro de la empresa, y crear en ellos la motivación necesaria para que puedan realizar sus labores de la mejor forma y sientan que son tomados en cuenta por la organización. Además de darle la oportunidad a los trabajadores de expresar su opinión en cuanto a la forma de realizar su trabajo, estimulando con esto la confianza y seguridad, a través de la puesta en práctica de acciones que impliquen sus conocimientos, habilidades y destrezas. A fin de mantener un clima confortable mediante la satisfacción de las necesidades de los trabajadores. 


\section{REFERENCIAS}

Chávez, E., y Vizcaíno, A. (2017). Talento humano: una contribución a la competitividad Organizacional. Mercados y Negocios: Revista de Investigación y Análisis. Volumen 1, N. 36, julio-diciembre 2017

Chiavenato, I. (2009). Comportamiento Organizacional. Editorial Mc Graw-Hill, Segunda Edición. México

Gutiérrez, C. (2012). Las empresas, el progreso y la mundialización. Reflexiones prospectivas. Venezuela. Universidad de Carabobo

Herrera, F. (2011). Evolución de las teorías sobre recursos humanos. México. EUMED

Hernando Vivar, M. (2014). Un modelo de control de gestión para la pequeña empresa familiar en España: especial referencia a Los activos intangibles. España. Universidad de Alcalá
Maslow, A. (1954) A theory of human motivation. PsycologicalReview

Moreno, M. (2008). Medición del Clima Organizacional de la pequeña y mediana empresa del sector manufacturero ubicada en Valencia-Edo. Carabobo. Trabajo Especial de Grado. Maestría en Ingeniería Industrial. Universidad de Carabobo, Bárbula

Saldarriaga, J. (2008) Gestión humana: tendencias y perspectivas. [Artículo en línea] Revista Estudios Gerenciales, vol. 24, núm. 107, abril-junio, 2008, pp. 137-159 Universidad ICESI. Cali, Colombia. Disponible

en: http://www.redalyc.org/pdf/212/2121 [Consultado: 14 de mayo de 2017]

Tamayo y Tamayo, M. (2007). El Proceso de la Investigación Científica. México: Limusa, Noriega 